\title{
Application of Pressure Probe and UV-MALDI-TOF MS for Direct Analysis of Plant Underivatized Carbohydrates in Subpicoliter Single-Cell Cytoplasm Extract
}

\author{
Yousef Gholipour, ${ }^{a}$ Hiroshi Nonami, ${ }^{b}$ and Rosa Erra-Balsells ${ }^{c}$ \\ a The United Graduate School of Agricultural Sciences, Ehime University, Matsuyama, Japan \\ b Plant Biophysics/Biochemistry Research Laboratory, Faculty of Agriculture, Ehime University, Matsuyama, \\ Japan \\ 'CIHIDECAR-CONICET, Departamento de Quimica Organica, Facultad de Ciencias Exactas y Naturales, \\ Universidad de Buenos Aires, Buenos Aires, Argentina
}

Single-cell cytoplasm sap (1-10 pL) was extracted by using a pressure probe glass microcapillary tip from tulip leaf and bulb and analyzed by UV-MALDI-TOF MS for free underivatized carbohydrate content. Three matrices including 2,5-dihydroxybenzoic acid (DHB), 2,4,6trihydroxyacetophenone (THAP), and carbon nanotubes (CNTs) in positive ion mode were selected for analysis because of acceptable carbohydrate-related signal reproducibility. Disaccharide and oligosaccharide (up to 15 Hex when THAP was used, 11 Hex with DHB, and 7 Hex with CNTs) were detected in tulip bulb cell cytoplasm sample. When DHB was used as matrix, neutral carbohydrates were more abundantly detected as sodiated cations; the sugar-related signals, however, appeared as dominant potassiated cations when THAP and CNTs were used. Small amount of monosaccharide was also detected in bulb cell cytoplasm with CNTs as matrix. UV-MALDI-TOF MS of leaf cell extract resulted in high-resolution detection of hexose and disaccharide with DHB, THAP, and CNTs. (J Am Soc Mass Spectrom 2008, 19, 1841-1848) (c) 2008 Published by Elsevier Inc. on behalf of American Society for Mass Spectrometry

$\mathrm{A}$ lthough suitable methods are available for analyzing plant metabolites via whole body or tissue extraction, which may provide general characterization of tissue components, biological investigations with single-cell resolution have their own pivotal position in biochemistry. They provide biological understanding about the cell in its natural condition and help analyze the cellular phenomena by accurate analysis of cell components. However, regarding very low volume of cell extracts and, subsequently, very low concentration of metabolites, such studies obviously need special high-resolution analytical instrumentations or techniques. After its application in biosciences, primarily to analyze proteins, a UV-MALDI MS technique was applied to analyze carbohydrates, particularly oligosaccharides, because of its high mass determination accuracy and high sensitivity [1-4]. Comprehensive reviews on applicability of UV-MALDI MS in different fields of carbohydrates chemistry and glycobiology can be found in the literature [5-8]. 2,5Dihydroxybenzoic acid (DHB), $\alpha$-cyano-4-hydroxycinnamic

Address reprint requests to Professor Rosa Erra-Balsells, CIHIDECARCONICET, Departamento de Quimica Organica, Facultad de Ciencias Exactas y Naturales, Universidad de Buenos Aires, Pabellón II, 3 P, Ciudad Universitaria, 1428-Buenos Aires, Argentina. E-mail: erra@qo.fcen.uba.ar acid (CHCA), 2,4,6-trihydroxyacetophenone (THAP) [5, 6], nor-harmane ( $9 \mathrm{H}$-pyrido[3,4-b]indole; $\mathrm{nHo}$ ) [9], carbon nanotubes (CNTs) [10, 11], and graphite [12] have shown applicability as matrices for UV-MALDI MS of neutral underivatized carbohydrates.

When a microcapillary glass tip enters a turgid plant cell, the positive turgor pressure originated from the stretched cell wall pushes the cytoplasm into the microcapillary. This phenomenon is the fundamental of pressure probe (PP) technique by which the turgor pressure of individual plant cells is measured [13]. Accordingly, microsampling of single-cell cytoplasm (in most cases, the vacuolar sap) [14, 15], followed by successful analysis of metabolites, particularly carbohydrates, by microfluorometric enzymatic assay has been developed [16-18].

The work reported here describes the successful use of UV-MALDI-TOF MS to analyze subpicoliter volumes of single-cell cytoplasm extracts obtained by using the PP technique, proposing appropriate matrices and sample preparation methods. Different matrices, including nHo, DHB, THAP, and CNTs, were examined for their efficiency for UV-MALDI of free underivatized carbohydrates from single-cell cytoplasm samples and benefits of their utilization are discussed. 


\section{Experimental}

\section{Materials and Preparations}

2,4,6-Trihydroxyacetophenone (THAP) was purchased from Fluka (St. Gallen Buchs, Switzerland), $\beta$-cyclodextrin, nor-harmane (nHo), and 2,5-dihydroxybenzoic acid (DHB) from Sigma-Aldrich (Tokyo, Japan), and multiwalled 10-30 nm carbon nanotubes (CNTs), mannitol, sucrose, and glucose from Wako Chemicals (Osaka, Japan). Tulip (Tulipa gesneriana L.) bulbs were supplied by a local grower.

Saturated solution of THAP in acetone was prepared. DHB and nHo solutions were prepared by dissolving $4 \mathrm{mg}$ of matrix in $2 \mu \mathrm{L}$ methanol/water 1:1 (vol/vol). CNTs were purified by successive rinsingcentrifuging with acetone and water/methanol, followed by suspending in water/methanol 1:9 ( $\mathrm{vol} / \mathrm{vol})$. Aqueous solutions of $10 \mu \mathrm{M}$ standard glucose, sucrose, and $\beta$-cyclodextrin were prepared, mixed with the matrix selected solution $(1: 3 \mathrm{vol} / \mathrm{vol})$, and deposited on the probe for external calibration.

\section{Single-Cell Cytoplasm Extraction}

The extraction instrumentation was a plant miniature PP equipped with a pressure transducer (model XTM190M-7-BAR-VG; Kulite Semiconductor Products, Inc., Leonia, NJ) [19-21]. Silicon oil is filled inside the microcapillary of the PP and also in the space between the capillary and the pressure sensor of the PP. When the capillary tip of the PP is inserted to a cell, a meniscus is formed by the boundary between the cell solution and the silicon oil in the capillary. A motorized metal rod of the PP can adjust the amount of volume in subpicoliter level so that the meniscus position can be manipulated accurately, because the silicon oil is incompressible under pressure applied by the movement of the metal rod inside the PP. The PP microcapillary tip (about 2.5 $\mu \mathrm{m}$ o.d.) was penetrated into the intact over-rested single bulb scale and leaf stalk cells, with cell volumes of about $1 \mathrm{~nL}$ and $5 \mathrm{pL}$, respectively (Figure 1). The pressure probe instrument and plant stand were placed on a magnetically floated vibration-free table (Magfloat; Sanaikogyo Co. Ltd.). Operation was monitored by an online stereomicroscopy system. To diminish probable cell-to-cell variation among samples when different matrices were used, the tip was penetrated into the same place with a bit more penetration depth or with a small relocation to enter the tip into an adjacent cell to extract the sample for the next matrix. Consequently, four samples were collected for four matrices from the same or very nearby punctured location. In addition, sampling was repeated five times from different locations on a $10 \times 10 \mathrm{~mm}^{2}$ area on the bulb surface and on a $2 \times 4 \mathrm{~mm}^{2}$ on the leaf stalk, to collect a total of 20 samples from bulb and 20 from leaf. Each experiment was repeated three times, using different bulbs all kept under similar conditions ( $80 \%$ to $90 \%$ relative humidity, $5{ }^{\circ} \mathrm{C}$ ). Turgor pressure caused the entering of $1-10 \mathrm{pL}$ (varying with cell size, tissue type, and cell water status) of cell cytoplasm into the microcapillary. The tip was then inserted into a micropipette containing $1 \mu \mathrm{L}$ of water followed by transfer of extract into the pipette by applying positive pressure in the microcapillary.

Sample preparation: In Method A, solution in the pipette was mixed in vitro with $3 \mu \mathrm{L}$ of matrix solution and $1 \mu \mathrm{L}$ of vortexed analyte-matrix mixture was deposited on a 64-well gold-covered probe (Applied Biosystems) and air-dried. Alternatively, in Method B, extract solution in pipette tip was immediately deposited on a layer of air-dried matrix on the probe, obtained from $1 \mu \mathrm{L}$ of the corresponding matrix solution/ suspension. Best results were obtained with DHB in Method A, and THAP and CNTs in Method B.

\section{UV-MALDI-MS Analysis}

For UV-MALDI-TOF MS analyses, delayed extraction in positive and negative ion mode was applied on the Voyager-DE STR time-of flight mass spectrometer (Applied Biosystems, Foster City, CA), equipped with a 337 $\mathrm{nm}$ nitrogen laser (3 ns pulse width). An accelerating potential of $20 \mathrm{kV}$ was used. Accumulated mass spectra from 200 shots per spot (plate well) and also accumulated mass spectra from different spots related to a particular matrix were recorded and compared (Figures 2, 3, 4, and 5). Mono- and disaccharide suspected peaks in cytoplasm extracts were compared with those from standard glucose and sucrose (Figures 2, 3, and 4). Sucrose and oligosaccharide peaks from cytoplasm extracts were analyzed by MS/MS (post-source decay mode (PSD); data shown in Supplementary Material, which can be found in the electronic version of this article. All the analyte MS data were compared with those of the corresponding matrix acquired under similar experimental conditions (Figures 2a-4a).

\section{Results and Discussion}

\section{Cytoplasm Microsampling}

Pressure probe facilitated microsampling and appeared to be a critical instrument for such studies; vibration-free micro-scale manipulation of microcapillary glass, and online monitoring of the operation by a stereomicroscope were quite necessary for precise and also repeated sampling from a predetermined location. On the other hand, a PP sensitive pressure transducer connected to a digital gauge provided observing the pressure change inside the microcapillary when the tip entered in the plant (i.e., factual penetration of tip into the cell could be confirmed by an instantaneous great decrease in PP pressure). Furthermore, a PP can provide managed negative pressure (which is reasonably necessary to extract sufficient sap when cells have insufficiently low turgor pressure) and positive pressure (to push the sap out of the microcapillary glass after extraction) inside the microcapillary glass.

A primary anatomical study helped select the location and penetration depth to acquire carbohydrates from 

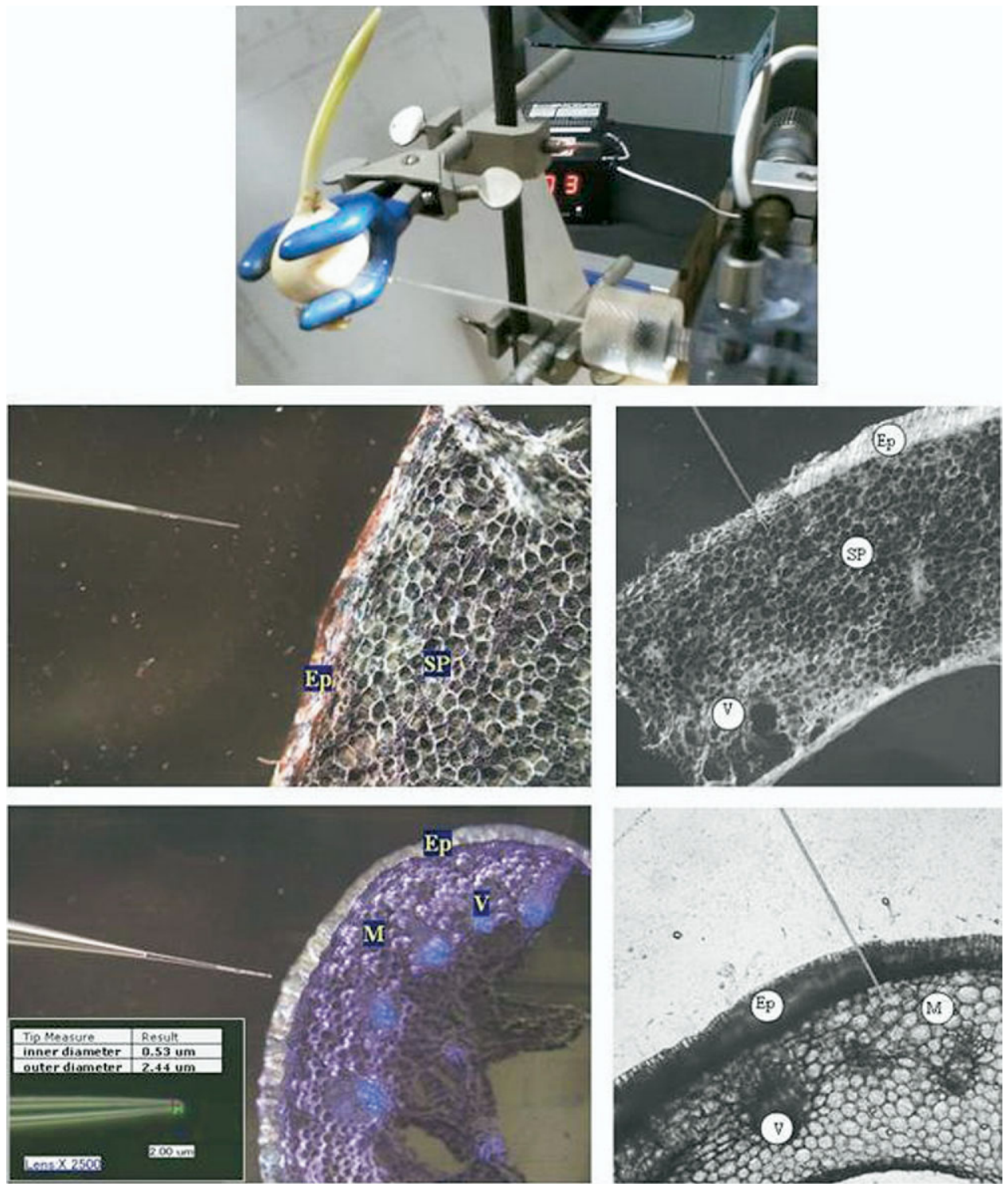

Figure 1. (Top) Microsampling of intact tulip bulb by pressure probe; (middle left) cross-section of bulb; (bottom left) leaf tissues demonstrating the anatomy of extraction locations of the plant material (dyed with Safranin 0.1\% and toluidine blue 0.1\%; photographed by Olympus DP50 florescence microscope in scanning mode). Inset located in the bottom photo shows the inner and outer diameters of the microcapillary tip used for current experiment (photographed and measured by Keyence VHX-900 digital microscope). SP and M: bulb storage parenchyma and leaf mesophyll cells, respectively, from which cytoplasm samples were extracted; Ep: epidermal layer; V: vascular cells. In the top photo, notice abundant dark blue granules (indeed starch granules stained with Lugol's solution) inside the cells regarded as typical mark of storage cells of the bulb; those cells occupy the major volume of the bulb; (middle and bottom right) trans-sections of bulb and leaf tissues to demonstrate the extraction location.

suitable parenchyma cells located in storage tissues of the bulb and mesophyll of the leaf (Figure 1, middle- and bottom-left). Accordingly, it was found that bulb storage parenchyma cells and leaf mesophyll parenchyma occupy most trans-section area of the bulb and leaf tissue, respectively. Except when the microcapillary tip rarely entered the vascular cells (which could be found easily by turgor pressure difference between vascular and regular parenchyma cells), storage parenchyma cells of the bulb showed acceptable similarity in their carbohydrate content; this was also true for mesophyll cells of leaf (Figure 1, middle and bottom right). The main variation between same cells was the signal intensity because of the difference in the volume of the extract and, consequently, of the amount of metabolite on the probe.

\section{Analysis of Carbohydrates in Leaf Cell Extract}

Almost no carbohydrate-related signal was detected in negative ion mode; accordingly, positive ion mode was 


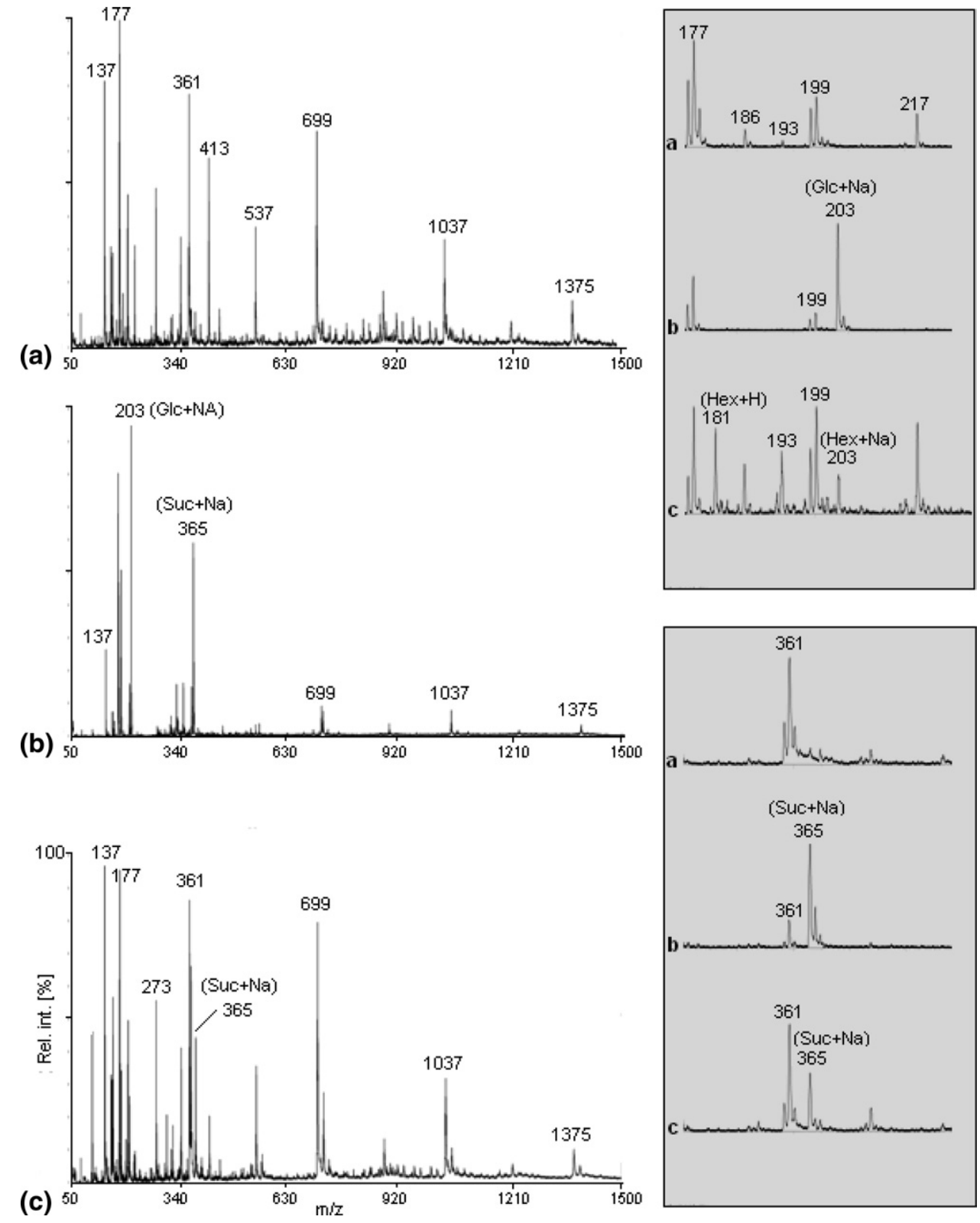

Figure 2. Positive ion mode UV-MALDI-TOF mass spectra of (a) DHB alone; (b) standard glucose and sucrose mixed with DHB (Method A); (c) leaf cytoplasm extract mixed with DHB (Method A). Insets magnify signaling range of $\mathrm{m} / \mathrm{z}$ related to hexose (top) and sucrose (bottom) from corresponding mass spectra on the left.

used for data acquisition and carbohydrate analysis because of its satisfactory signal reproducibility. Except for nHo (data not shown), the other three matrices could desorb/ionize neutral carbohydrates (DHB, Method A; THAP and CNTs, Method B).

Leaf PP microsampling was carried out from mesophyll parenchyma cells (Figure 1, bottom), which are actively involved in loading and transportation of carbohydrates, particularly sucrose. Although monosacchariderelated signals (in protonated, sodiated, and potassiated forms) were detected with DHB, THAP, and CNTs (Figures 2-4, insets), CNTs showed better adaptability to desorb/ionize monosaccharide from their surface with higher sensitivity, resolution, and $\mathrm{S} / \mathrm{N}$ ratio (Figure 4, inset). Unlike the case of bulb cytoplasm extract (see below), no significant distinct behavior of matrices for the type of yielded cations (whether sodiated or potassiated) was observed. However, all three common types of monosaccharide and disaccharide cation signals yielded more efficiently with THAP and CNT, using Method B for sample preparation. CNTs showed the best performance as matrix with high sensitivity and resolution as well as minor number of interfering signals (Figures 2a, 3a, and 4a). No oligosaccharide-related signal was detected in leaf samples. Similarly, oligosaccharide presence in tulip leaf cells could not be demonstrated by previous research 
(a)

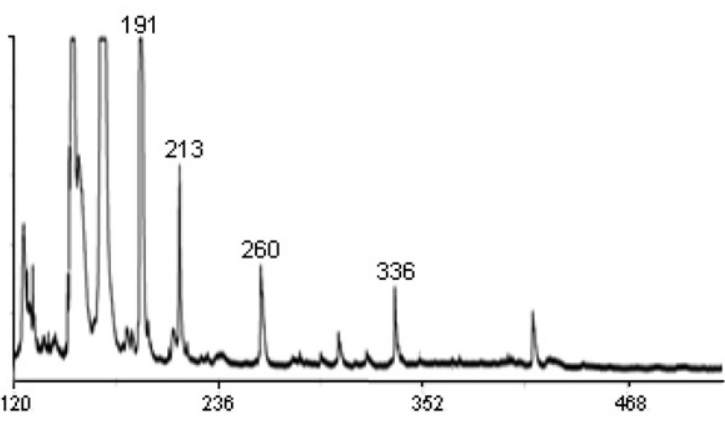

(b)

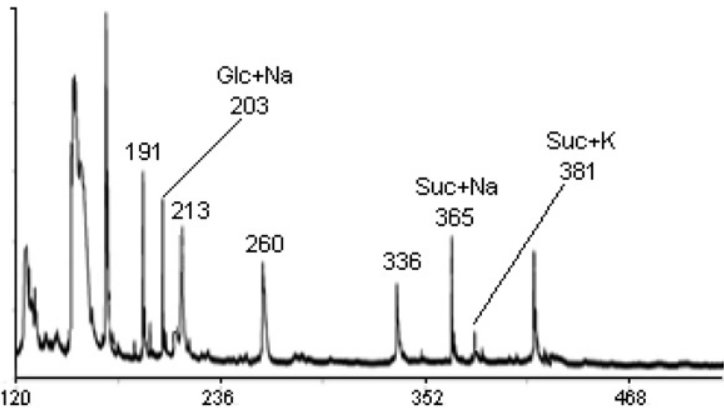

(c) 12

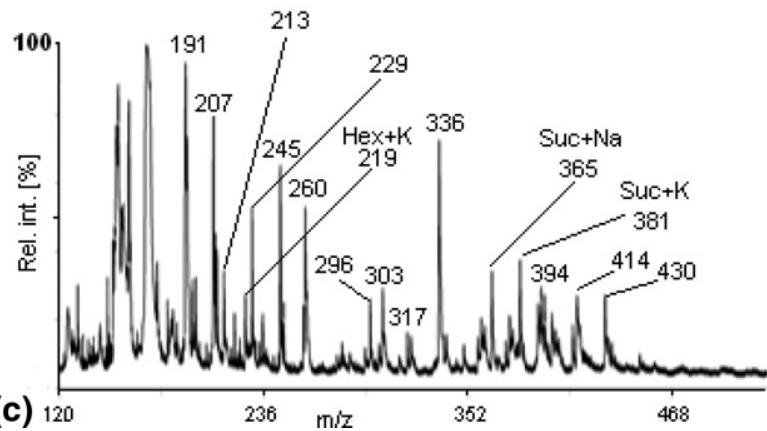

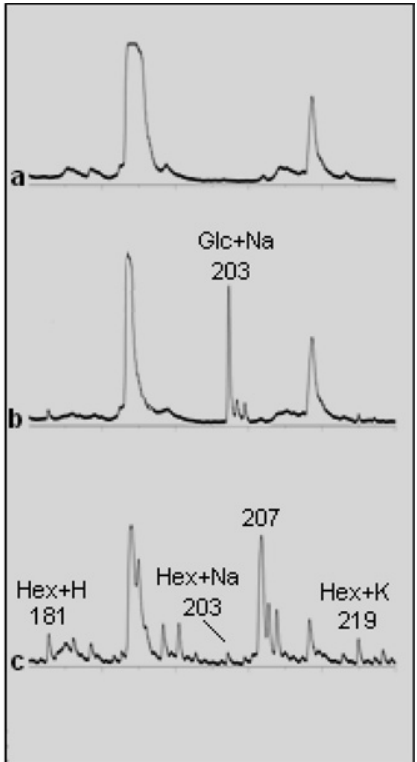

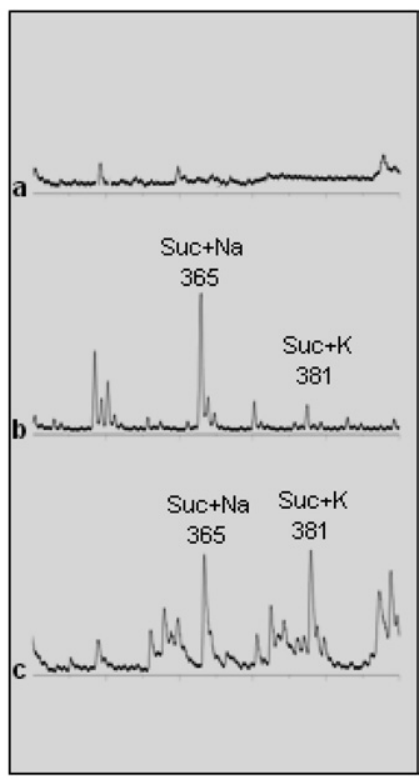

Figure 3. Positive ion mode UV-MALDI-TOF mass spectra of (a) THAP alone; (b) standard glucose and sucrose deposited on THAP (Method B); (c) leaf cytoplasm extract deposited on THAP (Method B). Insets magnify signaling range of $\mathrm{m} / \mathrm{z}$ related to hexose (top) and sucrose (bottom) from corresponding mass spectra on the left.

work, which used 2D-paper and thin-layer chromatography methods to analyze carbohydrate content of different tulip tissues, including leaf [22].

\section{Analysis of Carbohydrates in Bulb Cell Extract}

Application of DHB as matrix yielded sodiated di- and oligosaccharide cation signals in bulb cell cytoplasm samples; nevertheless, application of THAP and CNTs, as a direct result of less matrix-extract interaction and non-in vitro preparation accompanying with high abundance of native potassium ions, yielded dominant potassiated carbohydrates signals. Taking into account the anatomy of the bulb tissue trans-sections (Figure 1, middle-left) and regarding the PP microcapillary penetration depth led to microsampling from the cytoplasm (probably including vacuolar sap) of bulb carbohydratestoring parenchyma cells located immediately after the epidermal cell layer (Figure 1, middle right), where both insoluble and free carbohydrates are stored during rest period, detection of sucrose, fructo-oligosaccharides and, possibly, a small amount of monosaccharide, 
(a)
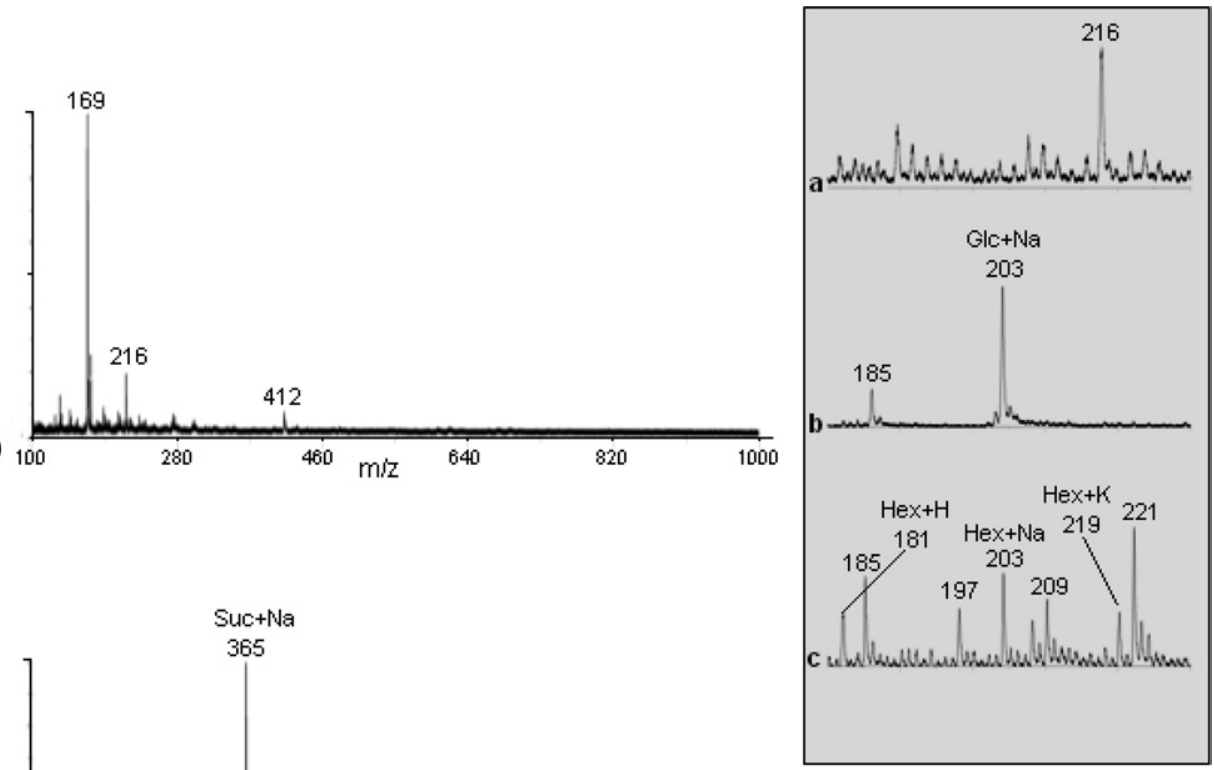

(b)
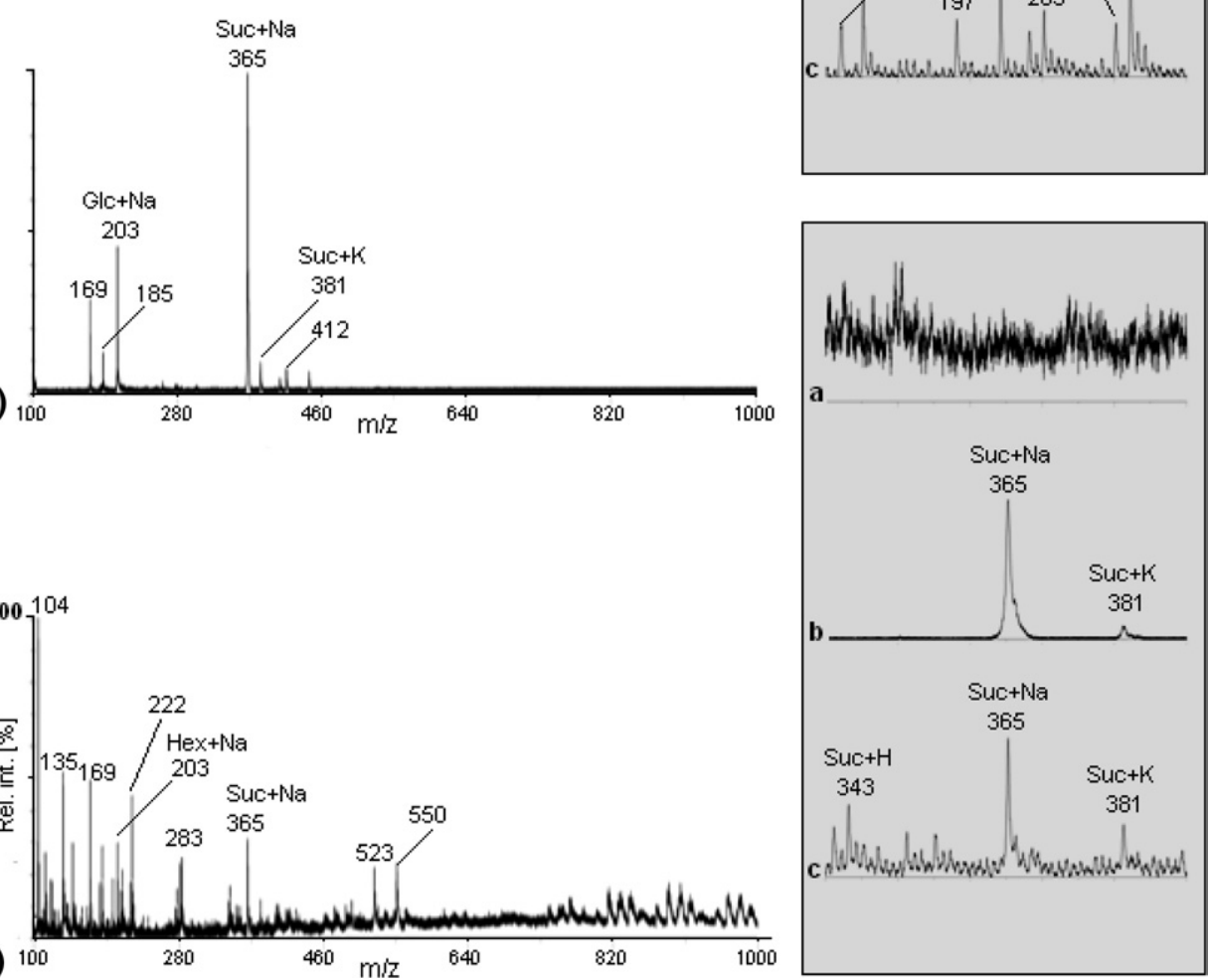

(c) 100

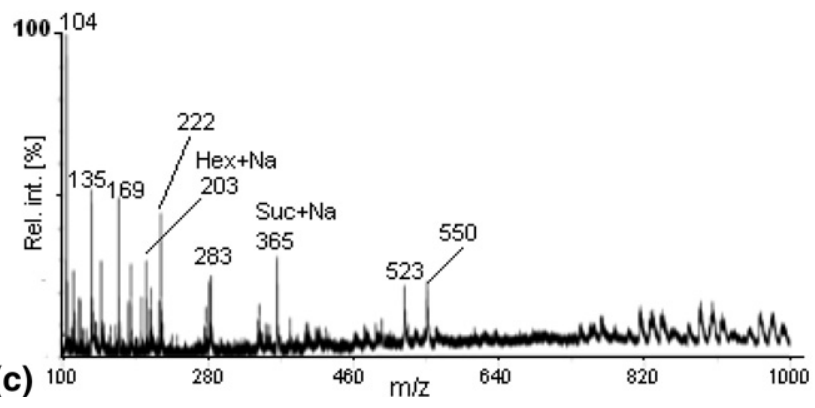

Figure 4. Positive ion mode UV-MALDI-TOF mass spectra of (a) CNTs alone; (b) standard glucose and sucrose deposited on CNTs (Method B); (c) leaf cytoplasm extract deposited on CNTs (Method B). Insets magnify signaling range of $\mathrm{m} / \mathrm{z}$ related to hexose (top) and sucrose (bottom) from corresponding mass spectra on the left.

was expected. Application of UV-MALDI-TOF MS technique successfully achieved the detection of disaccharide and fructans (up to 15 Hex when THAP was used, 11 Hex with DHB, and 7 Hex with CNTs) with satisfactory resolution (Figure 5). Least interfering signals accompanied by well-resolved peaks of disaccharide and 3 Hex oligosaccharide were acquired by using CNTs (Figure 5c). Nevertheless, CNTs failed in significant desorbing/ionizing of higher degree of polymerization fructans. Low-intensity signals related to monosaccharide were detected in bulb cell cytoplasm extract only when CNTs were used (data not shown).

\section{Conclusions}

Microprobing of single-cell cytoplasm by a PP glass microcapillary followed by UV-MALDI-TOF MS analysis showed adaptability for fast and accurate analysis and characterization of free underivatized carbohydrates in subpicoliter volumes of single-cell cytoplasm extracts from intact plants. Although neutral underivatized carbohydrates are difficult to ionize, utilization of three matrices including DHB, THAP, and CNTs resulted in efficient UV-MALDI of mono-, di-, and oligosaccharide as highly resolved sodiated and potassiated cations. The method demonstrated the possibility of 
(a)

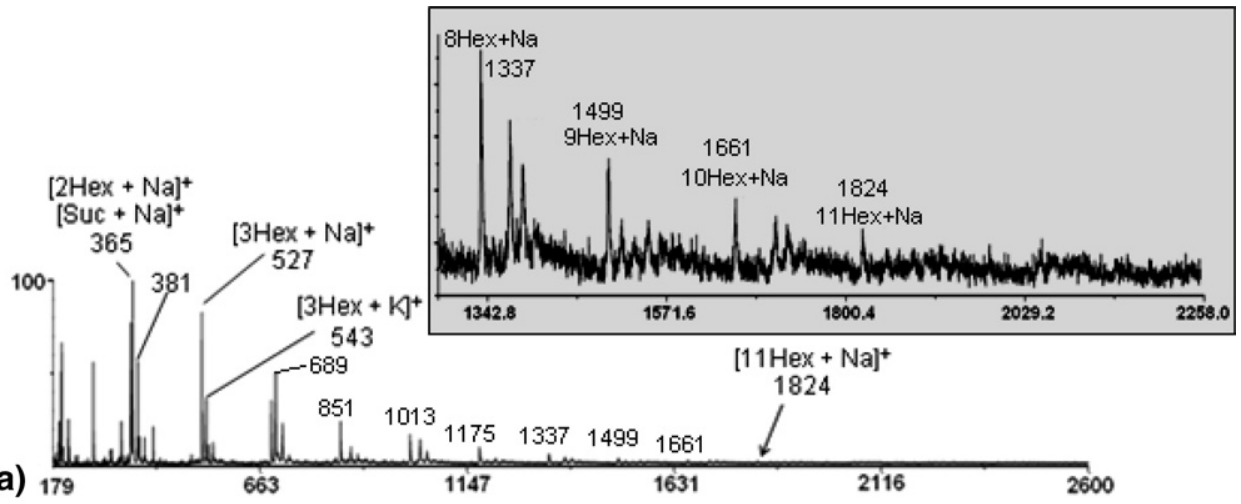

(b) 179
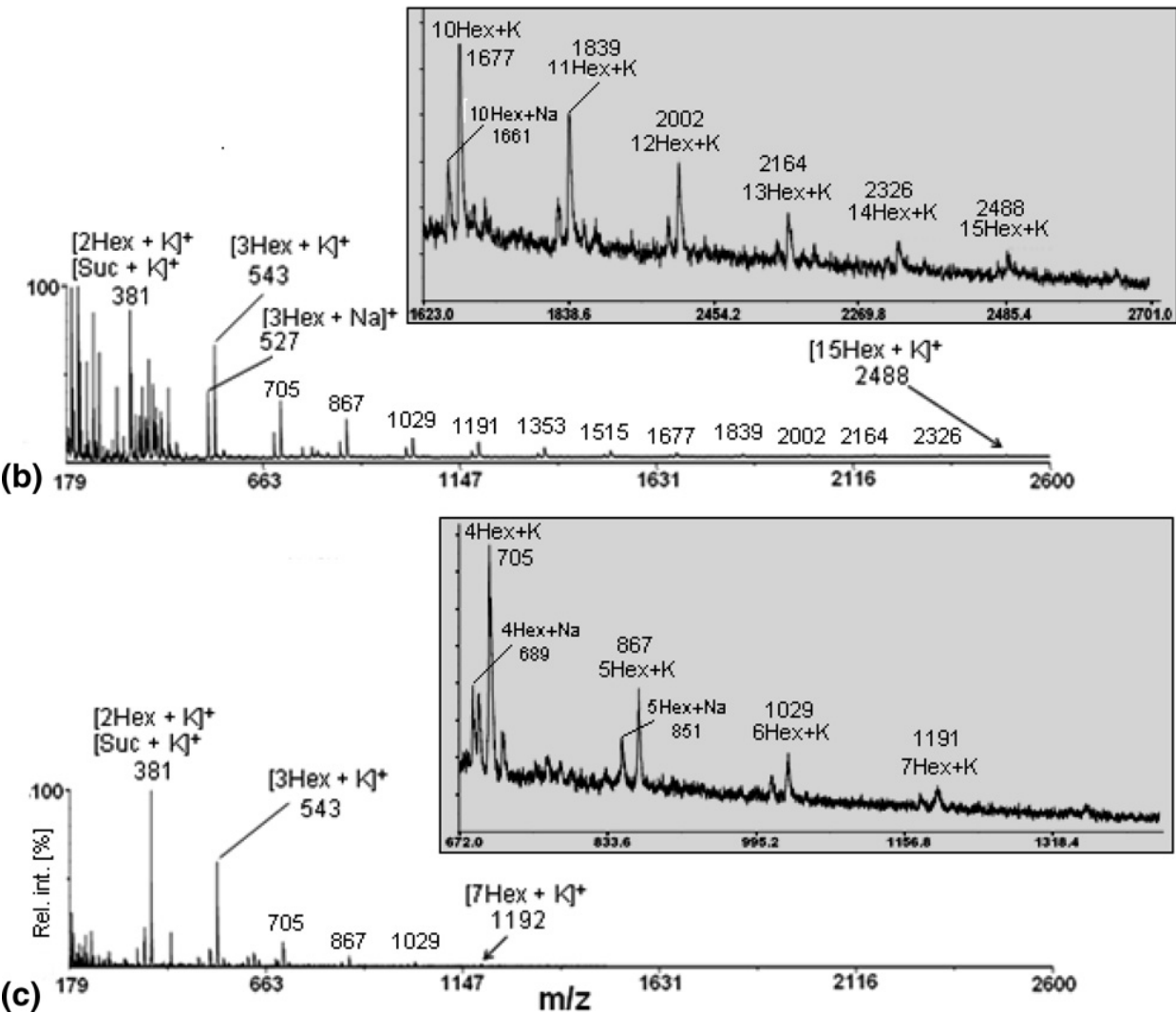

Figure 5. Positive ion mode UV-MALDI-TOF mass spectra of bulb cytoplasm extract using as matrix: (a) DHB (Method A); (b) THAP (Method B); (c) CNTs (Method B). Insets magnify $\mathrm{m} / \mathrm{z}$ range of fructans with highest degree of polymerization achieved with corresponding matrix.

time-resolved scanning of physiological events and biochemical reactions in an intact plant by direct characterization of metabolites during a whole growth and development period at the single-cell resolution level, particularly if the proper matrix and preparation methods are applied. On the other hand, analysis, especially with application of THAP and CNTs, which provided direct deposition of analyte on the matrix without the need for primary preparation of matrix-analyte mixture (sample preparation by Method B), yielded the best results and was quite fast and easy-to-do.

Finally, application of CNTs as a surface-desorbing matrix (Method B) demonstrated some interesting charac- teristics, including the lowest number of matrix signals at $m / z<400$ (Figures 2a-4a), efficient laser desorption/ ionization of carbohydrates, well-resolved peaks of carbohydrates with lower laser intensity, especially in positive mode, high $\mathrm{S} / \mathrm{N}$ ratio, and acceptable uniformity of signaling throughout of the spot on the plate. The lack of active functional groups in CNTs reduces matrix-analyte chemical interactions, and this fact would explain the uniformity of signaling. Thus, CNT properties as UVMALDI matrix depend on the purity of CNTs. Proper purification of CNTs helps to diminish some side problems such as abundant matrix signals or clusters in the low $m / z$ region of the spectra $(m / z<400)$. 


\section{Acknowledgments}

The authors acknowledge financial support for this work by the Japanese Government (Japanese Ministry of Education, Culture, Sports, Science, and Technology) scholarship program, Grant-inAid (S) from the Japan Society for the Promotion of Science (JSPS) for Scientific Research (20228004), UBA (X072), CONICET (PIP05/ 5443), and ANPCyT (PICT 06-0615). R.E.-B. is a research member of CONICET. UV-MALDI-TOF MS was performed as part of the Academic Agreement between R.E.-B. (FCEN-UBA, Argentina) and H.N. (CA-EU, Japan) with the facilities of the High Resolution Liquid Chromatography-integrated Mass Spectrometer System of The United Graduated School of Agricultural Sciences (Ehime University, Japan).

\section{References}

1. Mock, K. K.; Davey, M.; Cottrell, J. S. The Analysis of Underivatized Oligosaccharides By Matrix-Assisted Laser Desorption Mass Spectrometry. Biochem. Biophys. Res. Commun. 1991, 177, 644-651.

2. Stahl, B.; Steup, M. Analysis of Neutral Oligosaccharides by MatrixAssisted Laser Desorption/ Ionization Mass Spectrometry. Anal. Chem. 1991, 63, 1463-1466.

3. Harvey, D. J.; Naven, T. J. P.; Küster, B. Identification of Oligosaccharides By Matrix-Assisted Laser Desorption Ionization and Electrospray MS. Biochmem. Soc. Trans. 1996, 24, 905-912.

4. Stahl, B.; Linos, A.; Karas, M.; Hillenkamp, F.; Steup, M. Analysis of Fructans from Higher Plants by Matrix Assisted Laser Desorption/ Ionization Mass Spectrometry. Anal. Biochem. 1997, 246, 195-204.

5. Harvey, D. J. Matrix-Assisted Laser Desorption/Ionization Mass Spectrometry of Carbohydrates. Mass Spectrom. Rev. 1999, 18, 349-451.

6. Zaia, J. Mass Spectrometry of Oligosaccharides. Mass Spectrom. Rev. 2004, 23, 161-227.

7. Harvey, D. J. Analysis of Carbohydrates and Glycoconjugates by Matrix-Assisted Laser Desorption/Ionization Mass Spectrometry: An Update Covering the Period 1999-2000. Mass Spectrom. Rev. 2006, 25, 595-662.

8. Harvey, D. J. Analysis of Carbohydrates and Glycoconjugates by Matrix-Assisted Laser Desorption/Ionization Mass Spectrometry: An
Update Covering the Period 2001-2002. Mass Spectrom. Rev. 2008, 27, 125-201.

9. Nonami, H.; Fukui, S.; Erra-Balsells, R. $\beta$-Carboline Alkaloids as Matrices for Matrix-assisted Ultraviolet Laser Desorption Time-of-Fight Mass Spectrometry of Proteins and Sulfated Oligosaccharides: A Comparative Study Using Phenylcarbonyl Compounds, Carbazoles, and Classical Matrices. J. Mass Spectrom. 1997, 32, 287-296.

10. Xu, S. Y.; Li, Y. F.; Zou, H. F.; Qiu, J. S.; Guo, Z.; Guo, B. C. Carbon Nanotubes as Assisted Matrix for Laser Desorption/Ionization Timeof-Flight Mass Spectrometry. Anal. Chem. 2003, 75, 6191-6195.

11. Ren, S.; Zhang, L.; Cheng, Z.; Guo, Y. Immobilized Carbon Nanotubes as Matrix for MALDI-TOF-MS Analysis: Applications to Neutral Small Carbohydrates. J. Am. Soc. Mass Spectrom. 2005, 16, 333-339.

12. Zhang, H.; Cha, S.; Yeung, E. S. Colloidal Graphite-Assisted Laser Desorption/Ionization MS and $\mathrm{MS}^{\mathrm{n}}$ of Small Molecules. 2. Direct Profiling and MS Imaging of Small Metabolites from Fruits. Anal. Chem. 2007, 79, 6575-6584.

13. Hüsken, D.; Steudle, E.; Zimmermann, U. Pressure Probe Technique for Measuring Water Relations of Cells in Higher Plants. Plant Physiol. 1978, 61, 158-163.

14. Malone, M.; Leigh, R. A.; Tomos, A. D. Extraction and Analysis of Sap from Individual Wheat Leaf Cells: The Effect of Sampling Speed on the Osmotic Pressure of Extracted Sap. Plant Cell Environ. 1989, 12, 919-926.

15. Malone, M.; Leigh, R. A.; Tomos, A. D. Concentration of Vacuolar Inorganic Ions in Individual Cells Of Intact Wheat Leaf Epidermis. J. Exp. Bot. 1991, 42, 305-309.

16. Koroleva, O. A.; Farrar, J. F.; Tomos, A. D.; Pollock, C. J. Patterns of Solute in Individual Mesophyll, Bundle Sheath And Epidermal Cells of Barley Leaves Induced to Accumulate Carbohydrate. New Phytol. 1997, 136, 97-104.

17. Koroleva, O. A.; Farrar, J. F.; Tomos, A. D.; Pollock, C. J. Carbohydrates in Individual Cells of Epidermis, Mesophyll, and Bundle Sheath in Barley Leaves with Changed Export or Photosynthetic. Plant Physiol. 1998, 118, 1525-1532

18. Korolev, A. V.; Tomos, A. D.; Bowtell, R.; Farrar, J. F. Spatial and Temporal Distribution of Solutes In The Developing Carrot Taproot Measured at Single-Cell Resolution. J. Exp. Bot. 2000, 51, 567-577.

19. Nonami, H.; Boyer, J. S. Turgor and Growth at Low Water Potentials. Plant Physiol. 1989, 89, 798-804.

20. Nonami, H.; Boyer, J. S. Direct Demonstration of a Growth-Induced Water Potential Gradient. Plant Physiol. 1993, 102, 13-19.

21. Boyer, J. S. Measuring the Water Status of Plants and Soils, Chap. IV: Pressure Probe. Academic Press: San Diego, 1995.

22. Ho, L. C.; Rees, A. R. Aspects of Translocation of Carbon in the Tulip. New Phytol. 1975, 74, 421-428. 